\title{
Mesenchymal stem cells and immunomodulation: implications in bone tissue engineering
}

\begin{abstract}
Each year, millions of fractures occur all over the world, and a significant number of them carry the risk of failing to heal, resulting in delayed or non-union, requiring therapeutic intervention. Early immune response and inflammation after fractures plays an important role in the healing process; however, it has been shown that the prolonged inflammatory response is an inhibitory process in fracture repair. The exact mechanisms of inflammatory cells and their cytokines on fracture healing remains unclear. Some of the inflammatory mediators have been reported as encouraging factors in new bone formation, while others inhibit bone healing. From the past, stem cell therapy has always been an attractive option in bone tissue engineering for augmenting the fracture repair process. MSCs, as pluripotent stem cells, not only differentiate into pre-ostoblastic cells to form new bone, but they also apply immunomodulatory effects via a variety of mechanisms. This paper reviews the effects of immune cells and their cytokines on bone healing process and also discusses the most important modulatory mechanisms of MSCs on immune cells involved in bone tissue engineering.
\end{abstract}

Keywords: MSCs, immune system, inflammation, bone healing, tissue engineering
Volume 2 Issue 6 - 2016

\author{
Ahmad Oryan,' Nikolaos Papaioannou, ${ }^{2}$ \\ Amir Kamali,' loanna Stylianaki \\ 'Department of Pathology, Shiraz University, Iran \\ ${ }^{2}$ Department of Pathology, Aristotle University of Thessaloniki, \\ Greece
}

Correspondence: Ahmad Oryan, DVM, Department of Pathobiology, School of Veterinary Medicine, Shiraz, Iran. PO Box 7|345-|73|,Tel +98-7|3-6|3-8662, Email, oryan@shirazu.ac.ir

Received: August 30, 2016 | Published: September 23, 2016
Abbreviations: MSCs, mesenchymal stem cells; MHC, major histocompatibility complex; NK Cell, natural killer cell; IL-10, interleukin 10; IFN- $\gamma$, interferon gamma; TNF $\alpha$, tumor necrosis factor alpha; Th1, T helper 1 cells; Treg, T-cell inhibitory regulatory; Tlrs, toll-like receptors; PGE2, prostaglandin E2; NSAIDS, nonsteroidal anti-inflammatory drugs; DCS, dendritic cells

\section{Introduction}

Bone tissue engineering and regenerative medicine is a multidisciplinary and emerging field in biomedicine. It involves application of principles of engineering, materials science and cell biology to help in development of biological substitutes to regenerate, maintain or improve the function of injured bone tissues In this regard, using multipotent progenitor stem cells has found a special place in this field of science. Mesenchymal stem cells (a type of progenitor stem cells) have the capability to differentiate into a wide variety of cell lines, including osteocytes, chondrocytes and adipocytes. $^{2}$ Recently published investigations have shown that the allergenic MSCs pose immunomodulatory properties, leading to a considerable interest in utilizing these types of cells in therapeutic options in bone tissue engineering. ${ }^{3-5}$ Adipose-derived MSCs particularly are an attractive option in cell-based therapy because of their advantages over bone marrow-derived MSCs, including better potential for expansion and differentiation as well as easier isolation with fewer side effects. ${ }^{6}$ MSCs can evade host immune response by two major mechanisms: first, MSCs express low levels of MHC class I molecules which protects them from certain NK cell mechanisms of deletion and second, MSCs are completely devoid of MHC class II molecules and other co-stimulatory molecules (CD40, CD40L, CD80, and CD86) required for immune rejection.?

\section{Inflammation and natural fracture healing}

Fracture healing is a unique biologic process that begins with formation of a soft callus that is subsequently mineralized and remodeled. In the early phase of fracture healing, inflammatory cells play a critical role through release of specific chemical mediators to recruit and activate a variety of cell types including MSCs and induce their differentiation. ${ }^{8,9}$ Although inflammatory cells such as T cells, B cells and macrophages are critical in the bone healing process, many studies suggest that $\mathrm{T}$ cells and their cytokines such as IFN- $\gamma$ and TNF- $\alpha$, play an important role in inhibition of bone regeneration. ${ }^{10}$ It has also been emphasized that T-cells play a critical role in the maturation of osteoclasts. It has been shown that TNF- $\alpha$ can have pro-apoptotic effects on osteoblasts, and increased level of this proinflammatory cytokine can enhance destruction of bone tissue. ${ }^{11}$ In contrast, the role of some inflammatory mediators such as IL-10 in new bone formation and augmenting fracture healing has further been confirmed and it has been shown that IL-10 regulates bone resorption, and its absence causes osteopenia, bone fragility and malunions. ${ }^{12}$

In the second phase of inflammatory reactions (chronic phase), the role of macrophages in fracture sites should be highlighted. Macrophages are characterized into two major subtypes including macrophage type I and II. They have many important roles during bone healing process, from phagocytosis of necrotic cells, bacteria and hematoma, to the release of several important cytokines and growth promotive factors that accelerate the transition from the inflammatory stage to the fibroblastic or proliferative phase of bone healing. There are at least two inflammatory responses to exogenous cells or graft implantation. In the type I response the Th1 cells trigger macrophage type I cells, which results in occurrence of an inflammatory reaction 
and subsequent cell or graft rejection. On the other hand, in the type II response, Th2 cells trigger macrophage type II activity which is a remodeling reaction and results in cell or graft acceptance in the body. Overall, bone healing is a quite complex biological process and the current data in this field are rudimentary, so further studies are required to elucidate the roles of different inflammatory cells and their cytokines in fracture repair. ${ }^{13}$

\section{Immunoregulatory effects of MSCs in bone regeneration}

MSCs possess significant immunoregulatory effects on both the innate and adaptive immune systems. With regard to the innate immune system, more recent investigations have shown that allogeneic MSCs promote the polarization of monocytes into the macrophage type II phenotype, leading to enhanced acceptance of bone grafts. This phenotype differentiation induced by allogeneic MSCs may occur through the NF- $\mathrm{KB}$ and STAT-3 pathways. ${ }^{13}$ It has been shown that MSCs have direct immunomodulating properties by hampering the activation and proliferation of T cells (both CD4+ and CD8+) and also inducing generation of Treg cells.

MSCs can be polarized into a pro-inflammatory phenotype by TLR4 stimulation, whereas TLR3 stimulation can polarize MSCs into an immunosuppressive phenotype, and they are characterized as MSC1 and MSC2 respectively. ${ }^{14}$ MSC2 has a significant effect in reducing pro-inflammatory cytokine levels such as IL-6, TNF- $\alpha$ and IL-1 $\beta$ three days after fracture. This process results in an enhanced regeneration via inhibiting tissue injury and progression of fibrosis. It has been shown that not only the production of inflammatory cytokines including TNF- $\alpha$, IL-6, IL-12, and IFN- $\gamma$ by macrophages is significantly suppressed by MSCs via mediation of PEG2, but the production of anti-inflammatory cytokines such as IL-10 and IL-12 has also been increased by such a mechanism. ${ }^{15}$ MSCs also induce an anti-apoptotic effect which can accelerate the process of bone healing. It has been suggested that this effect is correlated especially with lower levels of TNF- $\alpha$ expression in the callus. ${ }^{16}$ Some in vivo and in vitro investigations also illustrated that MSCs can modulate the expression of NK cells and B cells and protect the bone healing process in the defect site. ${ }^{17,18}$

DCs, known as antigen presenting cells, differentiate from monocytes and secrete IL-12, which aids in differentiation of CD4+ T cells into Th1 cells. MSCs have been shown to impair the differentiation of DCs from monocytes, as well as their activity such as secretion of pro-inflammatory cytokines. ${ }^{19}$ It has been demonstrated that activated $\mathrm{B}$ cells mediate increased bone turnover during inflammatory states via preservation of humoral immunity. Early investigations have shown that MSCs have inhibitory effects on proliferation, maturation, and antibody secretion of the B cells in an active inflammation through cell cycle arrest in the G0/G1 phase and by the production of soluble factors. ${ }^{20}$ Thus it seems plausible that the MSCs' immunomodulatory effects on these cells would promote an osteogenic state and bone regeneration. The mechanism of MSCs in Immunomodulation of bone healing is illustrated in Figure1.

\section{Conclusion}

Inflammatory cells and their products (cytokines) play a critical role in bone healing. Along with growth promotive factors, inflammatory mediators also guide recruitment and differentiation of MSCs. Although inflammatory response is an important part of fracture repair, particularly during the early and remodeling phases of the healing process, long-term exposure to T cells, B lymphocytes and to inflammatory signaling has been shown to impair bone regeneration. The role of different inflammatory cells, their subtypes and products in bone regeneration is very complex and has not yet been fully clarified. Therefore, understanding the exact mechanisms of immune cells in the bone healing process and the precise routes to control them will be necessary to modulate inflammation as a new therapeutic option in bone tissue engineering and regenerative medicine. In this regard, MSCs can be effectively utilized due to their immunomodulatory effects on inflammatory cells which could change the functions and differentiation of immune cells in specific microenvironments (Figure 1).

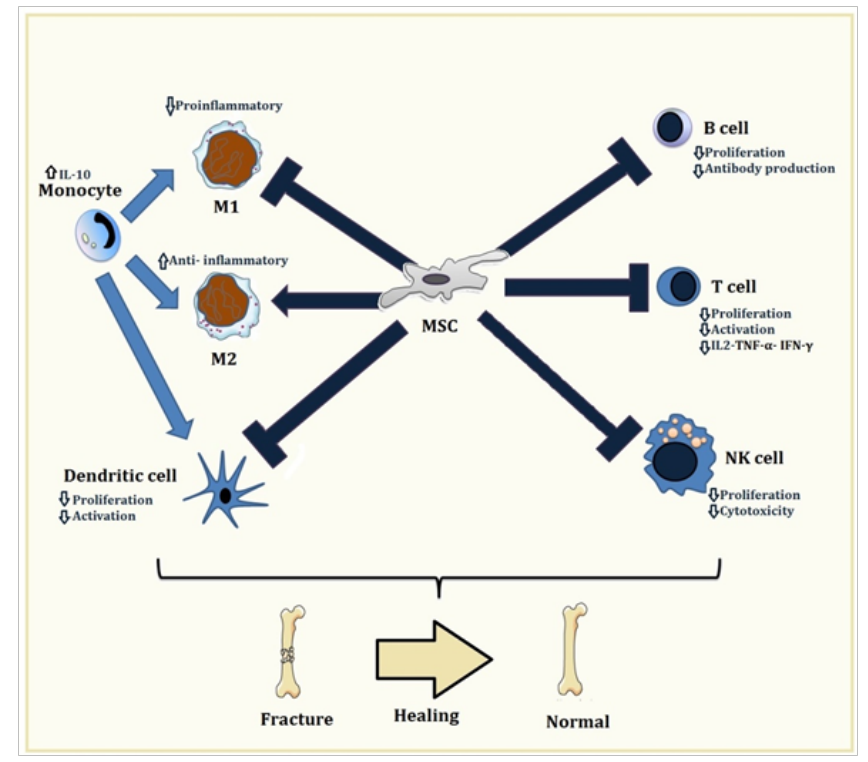

Figure I MSCs' immunmodulation of innate and adaptive immune cells in the bone healing process.

A key concern in using MSCs or other immunomodulators is that their negative regulation may cause an over-immunosuppression. Thus, great care should be taken to prevent systemic or local immunosuppression in patients, which could facilitate tumorigenesis or increased susceptibility to opportunistic infections. In general considering the current knowledge, it seems a very complex task to find out all synergisms and antagonisms among the various mechanisms contributing to the regulation of the immune response. Based on the above information, this article proposes the following areas for future investigations in this field: 1) developing new methods for analyzing the precise effect of inflammatory cells in bone fractures and investigating the effects of local and systemic delivery of various immunosuppressive factors (cytokine antagonists, corticosteroids and NSAIDS) on bone regeneration 2) pursuing the exact mechanistic aspects for inhibition of specific inflammatory cells and their effects on the bone healing process 3) investigating the effect of MSCs on immune cells and their subtypes in vitro and in vivo in order to discover the exact signaling pathways and molecules contributing to modulation of inflammatory response.

\section{Acknowledgements}

None. 


\section{Conflict of interest}

The author declares no conflict of interest.

\section{References}

1. Oryan A, Alidadi S, Moshiri A, et al. Bone regenerative medicine: classic options, novel strategies, and future directions. J Orthop Surg Res. 2014;9(1):18

2. Barry FP, Murphy JM. Mesenchymal stem cells: clinical applications and biological characterization. Int J Biochem Cell Biol. 2004;36(4):568-584.

3. Klyushnenkova E, Mosca JD, Zernetkina V, et al. T cell responses to allogeneic human mesenchymal stem cells: immunogenicity, tolerance, and suppression. J Biomed Sci Eng. 2005;12(1):47-57.

4. Krampera M, Cosmi L, Angeli R, et al. Role for interferon-gamma in the immunomodulatory activity of human bone marrow mesenchymal stem cells. Stem Cells. 2006;24(2):386-398.

5. Oryan A, Alidadi S, Moshiri A. Current concerns regarding healing of bone defects. Hard Tissue. 2013;2(2):13.

6. da Silva Meirelles L, Chagastelles PC, et al. Mesenchymal stem cells reside in virtually all post-natal organs and tissues. J Cell Sci. 2006;119(11):2204-2213.

7. Majumdar MK, Keane-Moore M, Buyaner D, et al. Characterization and functionality of cell surface molecules on human mesenchymal stem cells. J Biomed Sci. 2003;10(2):228-241.

8. Claes L, Recknagel S, Ignatius A. Fracture healing under healthy and inflammatory conditions. Nat Rev Rheumatol. 2012;8(3):133-143.

9. Oryan A, Monazzah S, Bigham-sadegh a. bone injury and fracture healing biology. Biomed Environ Sci. 2015;28(1):57-71.

10. Nam D, Mau E, Wang Y, et al. T-lymphocytes enable osteoblast maturation via IL-17F during the early phase of fracture repair. PloS One. 2012;7(6):e40044.
11. Feldmann M, Maini RN. Anti-TNF alpha therapy of rheumatoid arthritis: what have we learned? Annu Rev Immunol. 2001;19:163-96.

12. Sasaki H, Hou L, Belani A, et al. IL-10, but not IL-4, suppresses infectionstimulated bone resorption in vivo. J Immunol. 2000;165(7):3626-3630.

13. Abumaree MH, Al Jumah MA, Kalionis B, et al. Human placental mesenchymal stem cells (pMSCs) play a role as immune suppressive cells by shifting macrophage differentiation from inflammatory M1 to antiinflammatory M2 macrophages. Stem Cell Rev. 2013;9(5):620-641.

14. Carrion FA, Figueroa FE. Mesenchymal stem cells for the treatment of systemic lupus erythematosus: is the cure for connective tissue diseases within connective tissue? Stem Cell Res Ther. 2011;2(3):23.

15. Asami T, Ishii M, Fujii $\mathrm{H}$, et al. Modulation of murine macrophage TLR7/8-mediated cytokine expression by mesenchymal stem cellconditioned medium. Mediators Inflamm. 2013;2013:264260.

16. Kovach TK, Dighe AS, Lobo PI, et al. Interactions between MSCs and immune cells: implications for bone healing. $J$ Immunol Res. 2015;2015:752510.

17. Corcione A, Benvenuto F, Ferretti E, et al. Human mesenchymal stem cells modulate B-cell functions. Blood. 2006;107(1):367-72.

18. Spaggiari GM, Capobianco A, Becchetti S, et al. Mesenchymal stem cell-natural killer cell interactions: evidence that activated NK cells are capable of killing MSCs, whereas MSCs can inhibit IL-2-induced NK-cell proliferation. Blood. 2006;107(4):1484-1490.

19. Spaggiari GM, Moretta L. Interactions between mesenchymal stem cells and dendritic cells. Adv Biochem Eng Biotechnol. 2013;130:199-208.

20. Tabera S, Perez-Simon JA, Diez-Campelo M, et al. The effect of mesenchymal stem cells on the viability, proliferation and differentiation of B-lymphocytes. Haematologica. 2008;93(9):1301-1309. 